\title{
Resources Allocation and Sharing in Wireless Virtual Networks
}

\author{
Edvin J. Kitindi and ${ }^{1}$ Michael P.J. Mahenge ${ }^{2}$ \\ Solomon Mahlangu College of Science and Education, Sokoine University of Agriculture ${ }^{1}$ \\ Centre for Information and Communication Technology (CICT), Sokoine University of Agriculture ${ }^{2}$
}

\begin{abstract}
The rapid growth of the mobile devices and its services being experienced in a recent years brought both challenges and opportunities in the wireless network systems. The scarcity of the resources, high capital and operational costs are among the challenges facing the provision of the growing mobile services. The development of wireless network virtualization brought hope for the future networks to fulfil the users' demands. It allows different service providers to share the same physical infrastructure which in return, reduces both capital expenses (CAPEX) and operational expenses (OPEX). In this paper, the framework for wireless network virtualization is proposed. Also, the detailed description of the model based on the software define network (SDN) architecture is presented. On top of that we have proposed a load based resources sharing approach among the virtual networks (VNs). Our proposed approach has shown that, through sharing the excess resources, the VNs can significantly minimize unnecessary cost and utilize the available resources effectively.
\end{abstract}

Keywords: Virtual networks, Resource Block, Resource allocation and sharing, Cost minimization, Effective resource utilization

\section{INTRODUCTION}

Recently, there have been an explosive growing demand for mobile services from the users. The mobile users demand for high data rate, reliable service anywhere, and any time in all environments. These demands bring both challenges and opportunities to the wireless networks. Network resources scarcities, high CAPEX and OPEX among others are the challenges that hinder the provision of services to meet the booming demands. Network virtualization technology brought hope toward solving the aforementioned challenges to meet the growing users' demands.

Recently most research gives more attention on the network virtualization technique and it is a key area for network development. It enables multiple virtual networks to use the same network infrastructure while each operates similar to traditional way without understanding the underlying virtualization process. In addition each existing Virtual Network $(\mathrm{VN})$ can run its own network protocols and architecture which is completely different from other VN. In order to satisfy the mass of the new services running on the network, many research activities for innovation and improvement are needed.

The NV can be viewed as a process of sharing part or the entire network system in an optimized way. This implies that wireless network virtualization (WNV) can go beyond the physical network limits, forming the network without borders. It can realize the convergence of heterogeneous wireless networks which can incorporate various radio access networks (RAN) in providing integrated services to users.

Generally, wireless network is considered as an extension of the wired network, the same applies to WNV being considered as an extension from wired network virtualization. NV irrespective of whether wired or wireless environments, is viewed as splitting of the entire/part of system into different isolated sections. These sections are assigned to different parties holding certain corresponding functionalities while sharing the same physical network infrastructures . However, the distinctive properties of the wireless environment, such as time-various channels, attenuation, mobility, broadcast, and interference, makes the problem even more complicated .

Since physical resources among the VNs, then the resource allocation management is important. In recent years several works have been proposed based on the resource allocation and sharing in WNV . Most of this works adopted a fixed resource allocation scheme. Some proposed a dynamic resources allocation, while maintaining the conventional techniques . As a results VNs over-purchase physical resources from the infrastructure providers (InPs) to guarantee the services during the high traffic period. Hence, during low traffic period VNs waste the resources and incur unnecessary cost.

Hence, in this paper we propose a method that allows the VNs to share their purchased resources so as to ensure its effective utilization and reduce the cost of possessing extra resources which are not in use. That is, we propose a collaborative approach for multiple VNs which allows the VNs to pay for the used resources only and share the excess resources with other VNs in needy. The contribution of this work can be summarized as: 
Vol. 10, Issue 7, July 2021

DOI 10.17148/IJARCCE.2021.10703

- We introduces a load based resource sharing methods among the VNs, to ensure the effective resources utilization

- A collaborative cost sharing for the excess resources to the VNs is proposed to guarantee a cost-effective utilization of resources for multiple VNs.

This paper is organized as follows: Section II provides a WNV Framework, section III gives the system model, and a proposed collaborative sharing approach is explained in section IV. The evaluation and simulation setups are given in section V. Furthermore, in sections VI and VII we discuss the simulation results and conclusion respectively.

\section{WIRELESS NETWORK VIRTUALIZATION FRAMEWORK}

The framework for efficient resource utilization and cost reduction for the WNVs is given in this section. We divide our framework into three main parts; the physical infrastructure with limited resources which are abstracted and allocated to the VNs, the virtual resources pool which contains the virtualized resources and the control management capabilities, and the last part is the VNs which request the virtual resources from the InPs through Virtual Resources Controller (VRC) as described in Figure 1. Figure 1, shows several VNs requesting for the resources from the InPs. The network resource controller allocates the corresponding resources to the respective $\mathrm{VN}$ based on their requests.

Let consider $N$ number of VNs in a given system, the set for VNs can be indexed as $\left\{N_{l}, N_{2}, N_{3}, \ldots, N_{i}\right\}$. If each VN have $U_{i}$ users and the given InP have $K$ wireless channels (Resource Block) each having $c_{i}$ channel capacity, then the maximum capacity of a given InP is given by $C_{m}=\sum_{i=1}^{K} c_{i}$, and the corresponding channel power being $p_{i}$. Then, the total power for a given InP will be $P_{t}=\sum_{i=1}^{K} p_{i}$.

Since there are many InPs in the real environment then, if there are $M$ InPs, the total capacity $C_{T}$ and Total power $P_{T}$ available for the allocation will be given as $C_{T}=\sum_{j=1}^{M} \sum_{i=1}^{K} c_{j, i}$ and $P_{T}=\sum_{j=1}^{M} \sum_{i=1}^{K} p_{j, i}$ respectively, where by $c_{j, i}$ is the channel capacity for channel $i$ of $\operatorname{InP} j$ and $p_{j, i}$ is the channel transmission power for channel $i$ of InP $j$.

The resource pool in this case will contain the limited resources depending on the contribution from the InPs. The total resource block (RB) to be allocated to the VNs is limited by the available resources for each InPs. However, once the resources are allocated to the VNs, there will be a possibility for the VNs to share their allocated resources with each other through the control of the VRC which increases the effectiveness of the resource utilization, reduces cost of the resources and delay time of the users. Not only that, but also improves the quality of the services (QoS) to the users in the system. In this paper we focus on the effective resource utilization and the cost reduction.
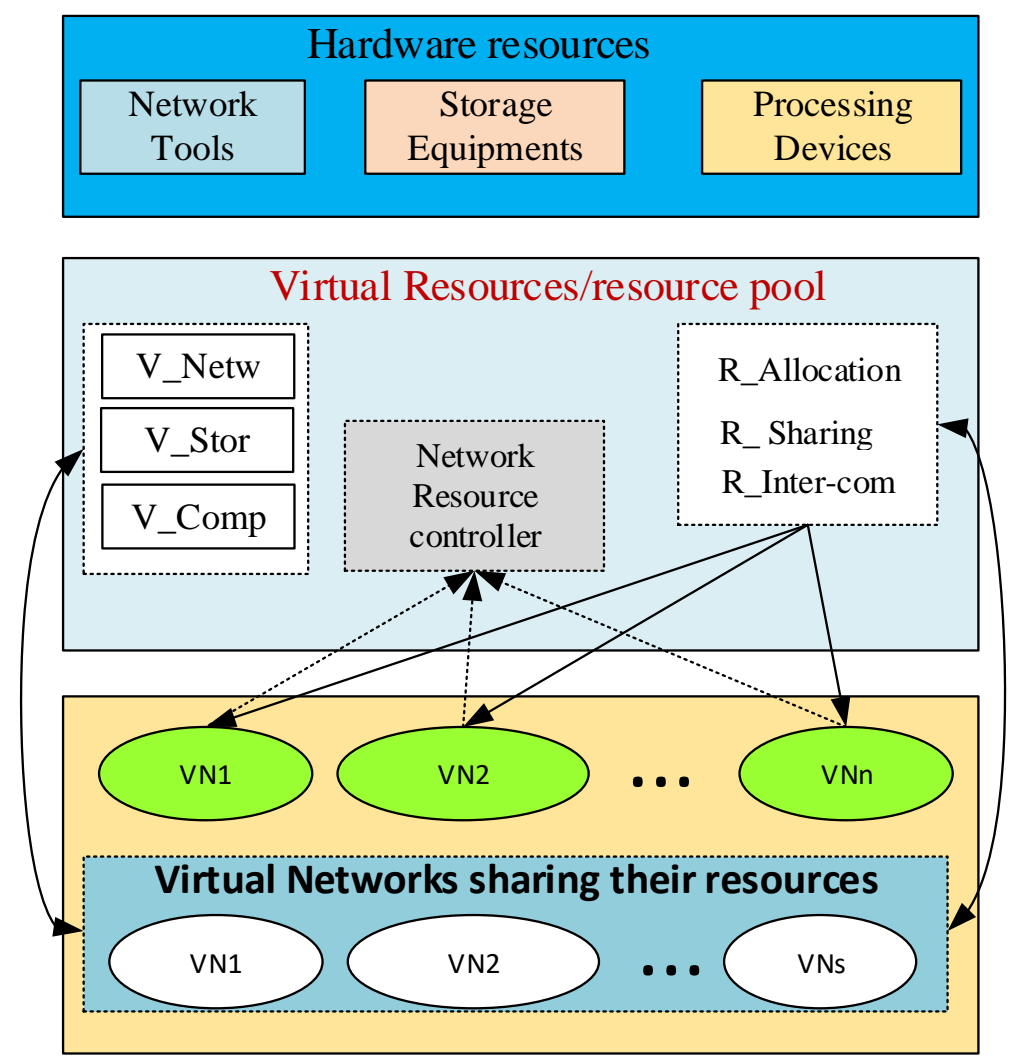

Figure 1: Framework of WNV 
Vol. 10, Issue 7, July 2021

DOI 10.17148/IJARCCE.2021.10703

\section{III.SYSTEM MODEL}

In our model we consider one InP with four VNs existing in the same geographical location, one eNodeB and the SDN based architecture have been adopted. The amount of the resource each VN request from the InP will depends on the traffic, with its readiness to pay for the resources. It should be noted that, there are three planes that form a basic components of communications system architecture. These are control plane, the data plane and the management plane. The control plane and management plane serves the data plane, which is responsible for transmitting the traffics the network carries. The management plane is considered a subset of the control plane which has administrative responsibilities in the system. Therefore we categories our model into two main parts, Control plane and Data plane as shown in Figure 2.

Control Plane: The control plane is in charge of integrating all the available RANs and resources together and slicing them into virtual resources. It mainly consists of network controller (NC) and Database. The NC is further subdivided into various components with different functionalities which are computation management (CM), Virtual Network Management (VNM), Resource Block Allocation (RBA), Inter-communication mobility management (ICMM) and Decision module (DM). All virtual networks are independent and there is no interference or conflict between them. Each consists of network infrastructure and wireless resources to be allocated to UEs.

- Network controller (NC) abstracts and splits the resources based on the requests, and then the abstracted resources are allocated to the corresponding virtual networks. The NC works together with the CM and the DM. CM is mainly responsible for calculating all parameters using data from database, data plane and then send them to the DM. The DM will determine whether parameters are met for the VNs to be allocated with the resources or share what they possesses. RBA will allocate the resources to the VNs based on the decision made by the DM. After the allocation then VNM manages all the VNs available while ICMM manages all the mobility of the resources among the VNs. It's the ICMM that responsible for determining the amount of the resources being shared among the VNs.

- Database: The abstract of whole network topology is stored here. This is a master resource database; it's the conceptual repository of all resource information in the network system's elements. The information includes for example the BSs (e.g. locations, transmit powers and physical resource block (PRB) usages) and UEs (e.g. Signal to Interference and Noise Ratio (SINR)) being collected from various data sources such as the one collected from the data plane.

Data Plane: The data plane is responsible for the transmission of user traffic via virtual networks. It composes heterogeneous network (HetNet) Base Stations (BSs) here refereed as eNodeB, which are responsible for forwarding and collecting information only. The UEs are direct linked with BSs to access the network system. The UEs ID is determined and the VN which it belong also are identified and forwarded to the control plane. To be specific the data plane incorporates the resources which links with the UEs directly, along with the necessary supporting resources for ensuring suitable virtualization, connectivity, security, availability, and quality [11].

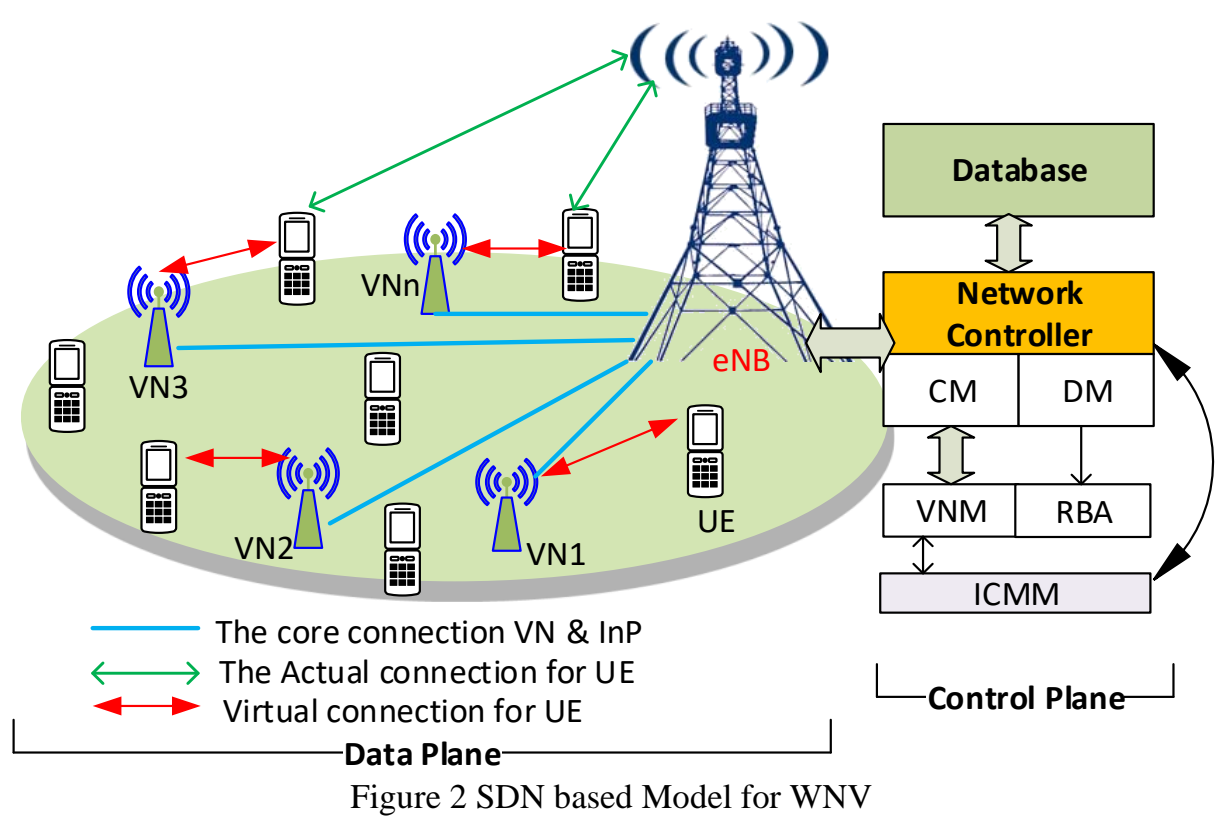


Vol. 10, Issue 7, July 2021

DOI 10.17148/IJARCCE.2021.10703

\section{IV.PROPOSED SHARING APPROACH}

The main purposes for the proposed approach is to increase the effectiveness of the resource utilization, and reduce unnecessary cost that the VNs can incur while increasing the number of users that can be served per given time. The proposed method will involves the following steps.

1. The UE send the request to their corresponding VN through eNodeB which will collect all the information about the UE ID, the VN it belong and the amount of the resources it request.

2. When the request submitted the $\mathrm{NC}$ will compute the load ratio for each VN through the CM to determine load for the given VN. The calculation will be based on the served request to the total request. Which will be given by:

$$
\rho_{i}=\frac{\text { Number of RB occupied by UE served by VN i }}{\text { Total number of System RB }}
$$

The $\rho_{i}$ will be used to determine the level of load belong to each $\mathrm{VN}$ by the DM. This will determine to what extend the given $\mathrm{VN}$ is in need of the extra RB. The value lies between 1 and 0 , that is $0 \leq \rho_{i} \leq 1$. We define three scenarios in our case as follow:

$$
\rho_{i}=\left\{\begin{array}{c}
\text { Highly in need of RB } 0.9<\rho_{\mathrm{i}} \leq 1 \\
\text { Moderate in need of RB } 0.5<\rho_{\mathrm{i}} \leq 0.9 \\
\text { Does not need extra RB } 0 \leq \rho_{\mathrm{i}} \leq 0.5
\end{array}\right.
$$

3. Once the value is found, the DM will make decision to be implemented by RBA. The VNM and ICMM will be processing all the information to trigger the sharing of all excess RB available in any of to those VN to serve the unserved users in the needy VNs.

A. Cost analysis for utilizing the resources

In this subsection we provide how our proposed method will improve the resources utilization and reduce cost to given VN. Not only that but we will show how it will help to serve the starving users being subscribed to one of the VN in the system. Let $\gamma, R B_{\text {Alloc }}, R B_{\text {Used }}$ and $R B_{\text {Excess }}$ be the cost of the using a unit RB per second, RB allocated to $\mathrm{VN} i$, RB used by $\mathrm{VN} i$ and unused RB by VN $i$ respectively, for $i=1,2, \ldots N$. Under fixed scenario the VN have to pay all the RB being allocated, it doesn't matter whether they have been utilized or not. If the proposed method applied in such situation the VN will reduce the cost of the resources accordingly and increase the number of the users to be served in the same system with the same resources.

$$
R B_{\text {Excess }}=R B_{\text {Alloc }}-R B_{\text {Used }}
$$

The loss $L$ the VN will get for unused $\mathrm{RB}$ will be given by

$$
L=\gamma R B_{\text {Excess }} * t
$$

Where $t$ is time in second. In our proposed approach the $L$ is termed as transferable loss. That is when the excess RBs are shares then, the $L$ is paid by other $\mathrm{VN}$ which is in need of the same. Based on (3) the cost is linearly proportional to the time which the VN possess the RBs.

The total cost for the VN is given in two form 1) when the VN doesn't share the excess RB it possess and 2) when the VN share the excess RB with the needy VN. Here the equation can be written as:

$$
\begin{aligned}
& T C 1=\gamma R B_{\text {Alloc }} * t+1 \\
& T C 2=\gamma R B_{\text {Used }} * t+1
\end{aligned}
$$

Equation (5) explains how the cost is shared among VN through sharing the RB based on the requirement. The $L$ in (4) will be distributed to other VNs who used the excess resources to serve their users. This reduces the unnecessary cost for possessing excess RB hence ensures the effective utilization of the available resources while serving the starved UEs. 
Vol. 10, Issue 7, July 2021

DOI 10.17148/IJARCCE.2021.10703

\section{EVALUATION AND Simulation SETUPS}

The evaluation was based on the total cost for each VN, the resources distribution among the VNs and its utilization. The simulation parameters are described in Table 1. We compare the simulation results for the proposed approach with the conventional approach. We assume that each user uses one RB at a time which implies that the number of connected users will not be more than the total RBs at the same time.

Table 1. System parameters for Simulation

\begin{tabular}{|l|l|}
\hline Parameters & Assumptions \\
\hline Number of VN & $4(\mathrm{VN} 1, \mathrm{VN} 2, \mathrm{VN} 3$ \& VN4) \\
\hline Number of channels K (RB) & $100(20 \mathrm{MHz})$ \\
\hline Number of RB for each VN & $24,23,28,25$ \\
\hline Number of Users in each VN & $17,32,21,30$ \\
\hline Running time & 10 Seconds \\
\hline
\end{tabular}

\section{VI.SIMULATION RESULTS}

In this section the simulation results for comparing the traditional method on cost and resource utilization which was obtained from the Matlab are presented. These results are based on the model and approach being explained in the previous sections. The parameters used for simulation are presented in Table 1.

\section{A. Effective resources Utilization}

Figure 3 and Figure 4 present the resource distribution among the VNs. In Figure 3 it can be observed that there are some VNs with excess resources while others have deficit of the RB to achieve its goal. For example VN 1 and VN 3 have extra RB being unused while VN 2 and 4 needs more resources to meet their requirements. Thus VN 1 and VN 3 waste the resources while others are in need of the same to use. On top of that it indicates that there are users not served by the VN 2 and VN 4 due to the lack of the RB. This led to the increase of waiting time for the user to be served. With the proposed approach Figure 4, shows that the VN is allocated with RB in need through sharing of the excess RB with others. That is the VN 1 and VN 3 shares the excess RB to the VN 2 and VN 4. Through this approach the resources are effectively utilized and more users can be served within short period of time.

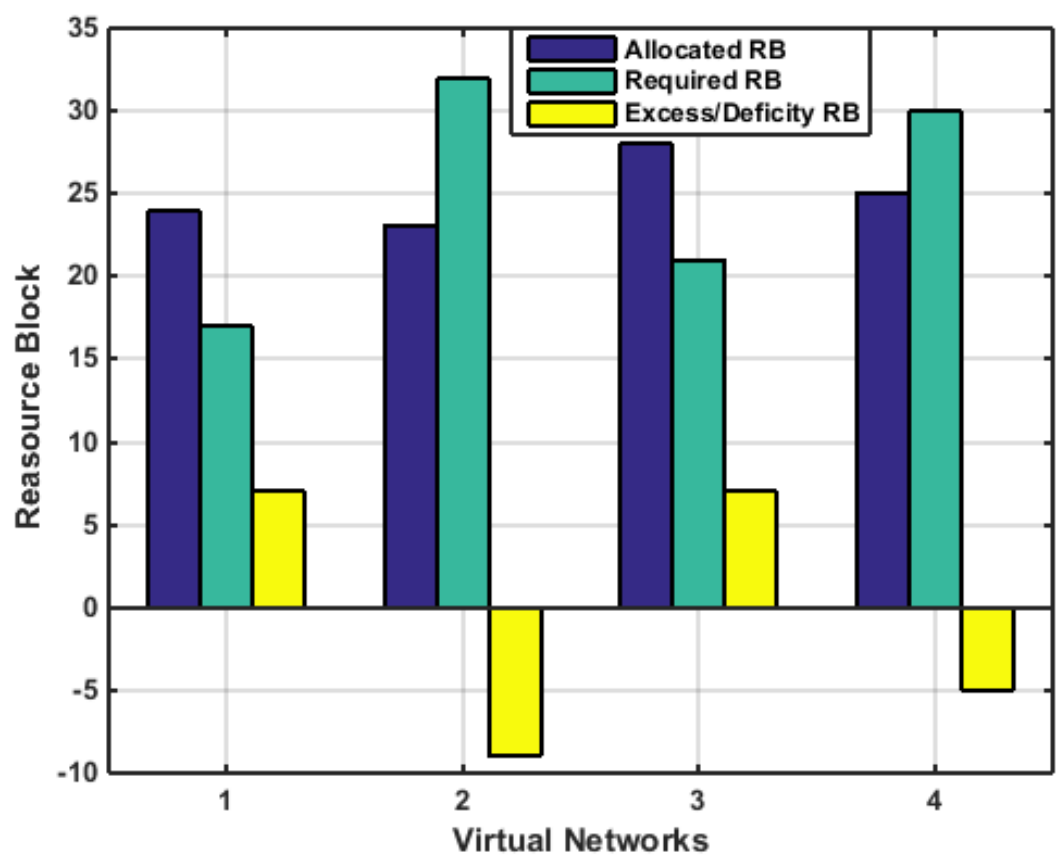

Fig. 3. Traditional RBs distribution among virtual Networks 
Vol. 10, Issue 7, July 2021

DOI 10.17148/IJARCCE.2021.10703

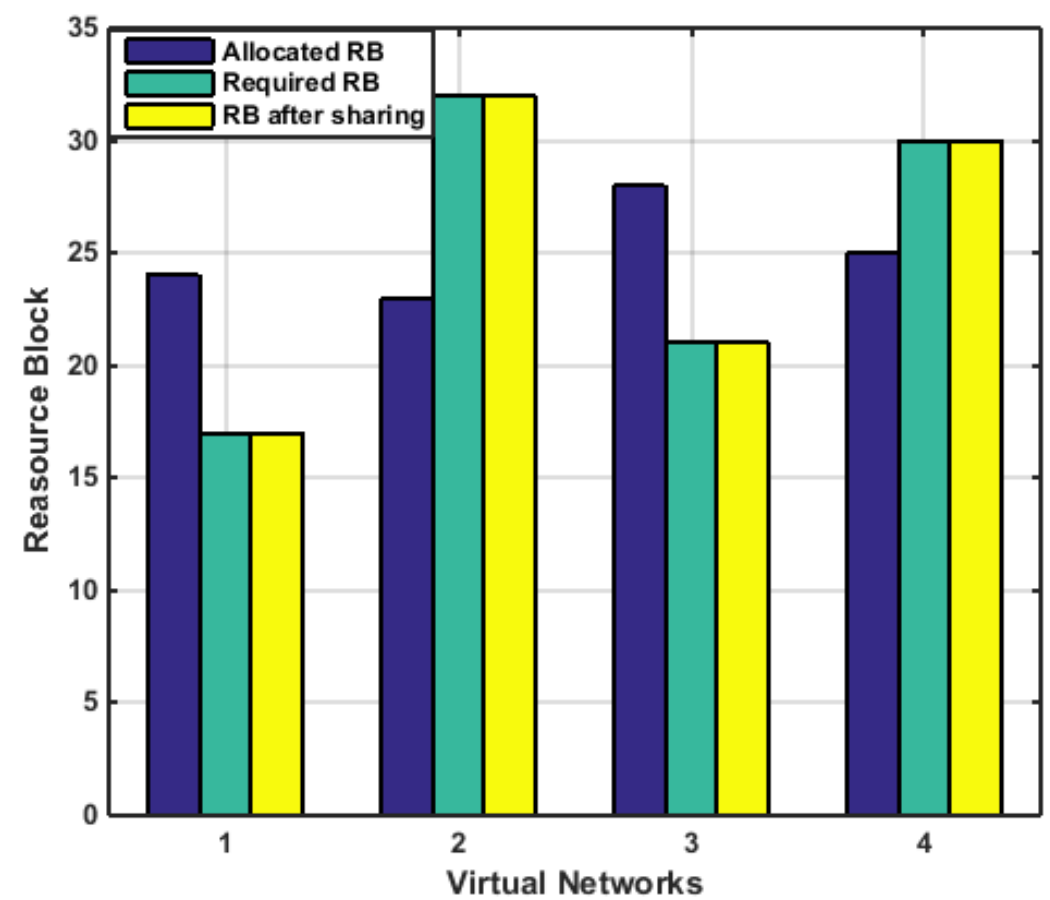

Fig. 4. Load Based RBs distribution among virtual Networks

B. Total cost for using the RBs in a given time

In conventional approach the VNs will pay the cost based on the resources allocated. It doesn't matter whether the resources have been used or not [9]. This increases the unnecessary cost for the VNs. To ensure QoS to the users, VN need to over purchase the resources. Figure 5 shows both the total cost for each VN when conventional approach is used and when a proposed approach is applied. It is clear that some VN pays unnecessary cost for the resources they possesses, for example VN 1 and VN 3 as can be seen in the Figure 5. However with our proposed approach there is a significant reduction of the total cost for the VN 1 and $\mathrm{VN} 3$ as they share their excess resources to the other VNs which are in need of it. The cost for the excess resources are being paid by the VNs that used it. Therefore with this method each VN will pay the corresponding cost of the RBs used.

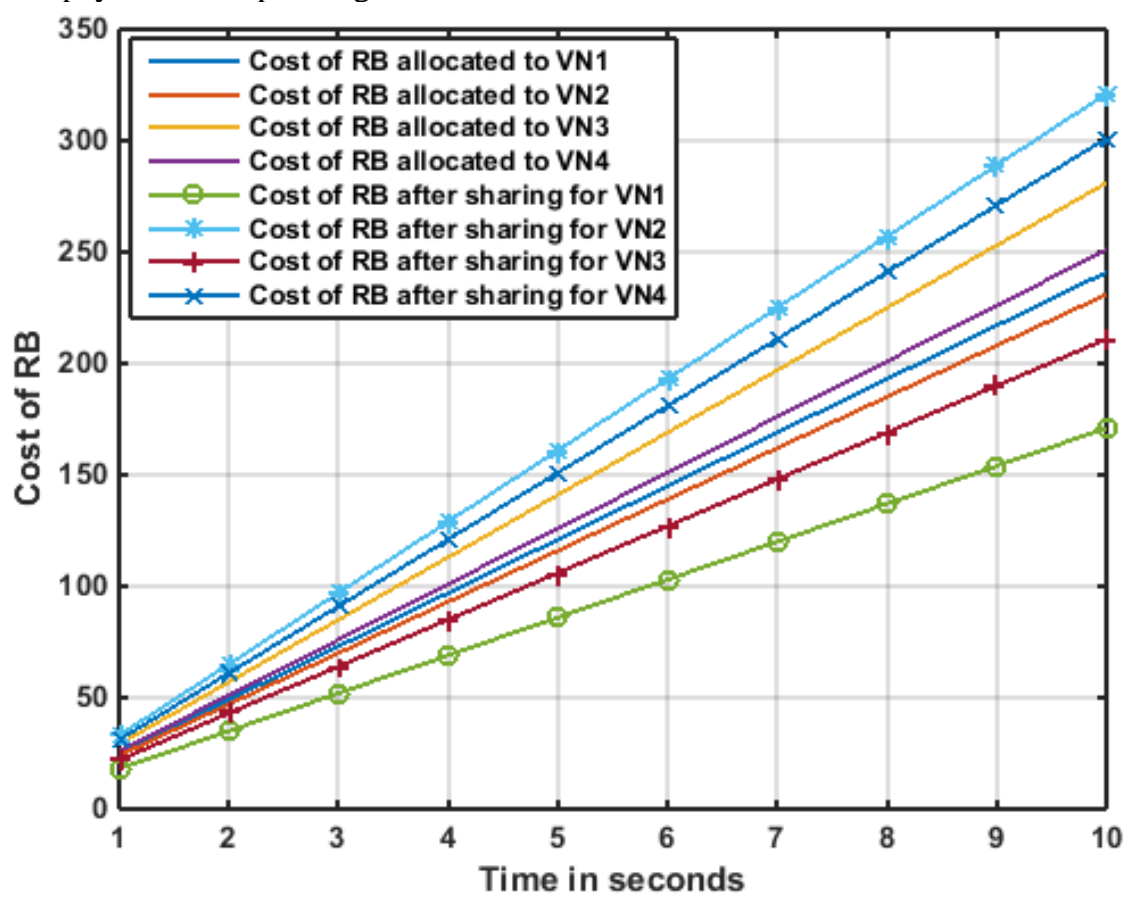

Figure. 5. Total cost for RBs based on conventional and Proposed approach 
Vol. 10, Issue 7, July 2021

\section{DOI 10.17148/IJARCCE.2021.10703}

\section{CONCLUSION}

In this paper, we proposed a load based resources sharing approach in virtual wireless networks. The key challenge for the proposed approach was to allow the sharing of the unused resources and reduce unnecessary cost that the VNs pays for excess resources. Our proposed approach managed to overcome such challenge through sharing the excess resources possessed among the VNs hence minimizing the unnecessary cost. The main features of the proposed algorithm are the ability to detect the unused resources and distributing it among VN which in return share the cost incurred. We have demonstrated that the VNs pays the cost of the resources used. In future we will focus on how the proposed approach will improve the quality of services by investigating the delay time and the data rate that the UEs will be experiencing. Also we will consider more than one eNodeB and InPs for the investigation.

\section{REFERENCES}

[1] H. Wen, P. K. Tiwary and T. Le-Ngoc; Wireless Virtualization, Springer, 2013.

[2] C. Liang and F. Yu, "Wireless Network Virtualization: A Survey, Some Research Issues and Challenges," IEEE Commun. Surveys \& Tutorials, pp. 358-380, 2015.

[3] Y. Han, X. Tao, X. Zhang and S. Jia, "Hierarchical Resource Allocation in Multi-Service Wireless Networks With Wireless Network Virtualization," IEEE Transactions on Vehicular Technology, vol. 69, no. 10, pp. 11811 - 11827, 2020.

[4] X. Wang, P. Krishnamurthy and D. Tipper, "Wireless network virtualization," in Int'l Conf. on Computing, Networking and Commun. (ICNC), San Diego, 2013.

[5] R. Kokku, R. Mahindra, H. Zhang and S. Rangarajan, "NVS: A Substrate for Virtualizing Wireless Resources in Cellular Networks," IEEE/ACM Transactions on Networking, vol. 20, no. 5, p. 1333 - 1346, 2012.

[6] Y. Zaki, L. Zhao, C. Goerg and A. Timm-Giel, "Lte wireless virtualization and spectrum management," in Wireless and Mobile NetworkingConference (WMNC), 2010.

[7] D. Yun and Y. Yi, "Virtual network embedding in wireless multihop networks," in 6th International Conference on Future Internet Technologies, New York, 2011.

[8] F. Fu and U. Kozat, "Wireless network virtualization as a sequential auction game," in INFOCOM, 2010.

[9] K.-m. Park and C.-k. Kim, "A framework for virtual network embedding in wireless networks," in 4th International Conferenceon Future Internet Technologies, New York, 2009.

[10] M. Yang, Y. Li, J. Liu , D. Jin, J. Yuan and L. Zeng, "Opportunistic Spectrum Sharing for Wireless Virtualization," in Wireless Communications and Networking Conference (WCNC), 2014.

[11] R. Xie, J. Wu, R. Wang and T. Huang, "A game theoretic approach for hierarchical caching resource sharing in 5G networks with virtualization," China Communications, vol. 16, no. 7, pp. 32 - 48, 2019.

[12] ONF, "SDN architecture," June 2014. [Online]. Available: https://opennetworking.org/wpcontent/uploads/2013/02/TR_SDN_ARCH_1.0_06062014.pdf. [Accessed 28 June 2021].

[13] X. Ting, P. Zhiwen, L. Nan and Y. Xiaohu, "Inter-operator resource sharing based on network virtualization," in International Conference on Wireless Communications \& Signal Processing (WCSP), Nanjing, 2015.

[14] X. Ting, P. Zhiwen, L. Nan and Y. Xiaohu, "Inter-Operator Resource Sharing Based on Network Virtualization," in Inter Conference on Wireless Communications \& Signal Processing (WCSP), 2015. 